\title{
Developing design-oriented strategies to combat regional scale climate change
}

\author{
B. Stone, Jr \\ Georgia Institute of Technology, City and Regional Planning Program, \\ USA
}

\begin{abstract}
This paper presents a study of residential parcel design and surface heat island formation in a major metropolitan region of the southeastern United States. Through the integration of high-resolution multispectral data $(10 \mathrm{~m})$ recorded by the National Aeronautical and Space Administration (NASA) with property tax records for over 100,000 single-family residential parcels in the Atlanta, Georgia metropolitan region, the influence of the size and material composition of residential land use on an indicator of surface heat island formation is reported. In contrast to previous work on the urban heat island effect, this study derives a parcel-based indicator of surface warming to permit the impact of land use planning regulations governing the density and design of development on the excess flux of heat energy to be measured. The results of this study suggest that the contribution of individual land parcels to regional surface heat island formation could be reduced by approximately $40 \%$ for average sized parcels through the adoption of specific land use planning policies, such as zoning and subdivision regulations, and with no modifications to the size or albedo of the residential structure.

Keywords: climate change, urban heat island effect, land use, urban planning.
\end{abstract}

\section{Introduction}

The significance of land use to climate change has only recently begun to receive serious attention within the technical literature. As reported by Kalnay and Cai [1], analyses of both surface and atmospheric temperature trends over the last half century suggest that land use change may be responsible for as much as $50 \%$ of the observed reduction in the mean diurnal temperature range - a phenomenon 
largely attributed to increasing minimum temperatures throughout the continental United States. Two principal causes of this increase in minimum temperatures, agriculture and the urban heat island effect, are identified as significant drivers of ongoing changes in climate. In short, an increase in surface temperatures resulting from land cover changes, independent of ongoing increases in greenhouse gas concentrations, appears to be a significant driver of climate change. In a similar vein, Foley et al [2] conclude from a global analysis of land use that while "[1] and use has generally been considered a local environmental issue ... it is becoming a force of global importance" (p. 570).

Despite a growing recognition of the importance of land use to climate change, the suite of mitigation strategies embodied in the still-evolving Kyoto framework remain almost entirely technological in nature, with little attention to strategies rooted in the design of human settlements. Because the process of climate change is driven by two distinct mechanisms - an increase in the heat absorbing potential of the atmosphere and an increase in surface heat emissions - there is a critical need to focus not only on greenhouse gas reductions but also on strategies to reduce surface heat emissions at the local level.

To address this issue, this article presents the results of a study of residential parcel design and surface warming patterns in the Atlanta, Georgia (USA) metropolitan region. With the aid of high resolution thermal infrared (IR) imagery and a parcel level geographic information system, the thermal properties of over 100,000 single family parcels are associated with the spatial structure and material composition of residential development throughout a $48 \mathrm{~km}^{2}$ study region. In measuring the thermal properties of individual land parcels, an "emissivity adjusted" indicator of the parcel black body flux is developed to account for the influence of variable surface emissivity and sky radiation captured in the thermal IR imagery. Furthermore, the predevelopment radiant flux is estimated and subtracted from the observed, post-development radiant flux to differentiate the warming impacts of urban development from natural, background sources of surface heat.

\section{Background}

In order to associate remotely sensed thermal IR data with land use planning policies it is essential that a "policy-relevant" indicator of surface warming be developed. While remote sensing technologies can provide a valuable tool for assessing the thermal performance of urban environments, these must be used with great caution to yield reliable information for land use planning. Most importantly, there is a critical need to isolate the influence of specific classes of land use on surface warming patterns. While previous work has demonstrated a clear distinction between the thermal properties of "urban" and "rural" land cover types, each of these broad categories of development is composed of a number of specific land use classes that are subject to an array of development regulations. If we are to mitigate heat island formation through the modification of specific zoning and subdivision regulations, we must be able to isolate the influence of these individual policies on surface warming. To be policy-relevant, 
an indicator of surface warming must be capable of quantifying the thermal characteristics of distinct and legally defined classes of land use.

In this section of the paper, I discuss three criteria that must be satisfied to derive a policy-relevant measure of heat island formation. These include: 1) the measurement of surface warming at the level of the individual land parcel; 2) the adoption of a flux-based rather than a temperature-based indicator to capture the effects of land area on warming; and 3) the adjustment of the radiant flux to more closely correspond to the sensible flux of heat.

\subsection{Measuring thermal performance at the parcel level}

In assessing the surface thermal properties of urban and rural land covers, many studies have adopted a unit of analysis consistent with the resolving dimension of a satellite radiometer, such as $1.1 \mathrm{~km}$ or $120 \mathrm{~m}$ (e.g., Roth et al [3]; Nichol [4]). An important limitation of adopting such a spatially uniform unit of analysis, however, is that it fails to conform to the irregularly shaped boundaries of individual land parcels, the unit of area at which land use is controlled in the United States. For example, in dense and well-mixed urban districts, a spatially uniform unit of analysis is likely to capture both commercial and residential land uses, confounding any attempt to isolate the thermal properties of a single land use class. Such an outcome may be categorized as a special instance of mixed pixel error - mixed, that is, in terms of land use class rather than land cover type.

The limitation of this type of mixed pixel error for planning analysis is that it obscures the underlying policy drivers of environmental performance. If we are interested in evaluating the environmental impacts of a particular planning policy, such as maximum impervious area regulations, we must first identify those zones subject to the policy. For example, within a square kilometer of urban land there is likely to be a number of unique land use classes, each with its own zoning regulations governing the permissible area of impervious cover per parcel. In measuring the environmental characteristics of an area of land subject to multiple zoning regulations, it is difficult to assess the relative influence of any single policy on environmental performance. For the purposes of planning research, a more methodologically sound approach entails the measurement of surface warming at a dimension compatible with land use regulation, such as a zoning district or individual land parcel. As adopted herein, a parcel-based approach to surface warming analysis permits parcel-specific land use policies, such as zoning regulations, subdivision regulations, and building codes, to be associated with thermal performance at the same spatial dimension.

\subsection{Accounting for the effects of land area on surface warming}

While parcel-based measures of surface warming are needed to associate heat island formation with land use policies, the adoption of a spatially irregular unit of analysis complicates the use of surface temperature as an indicator of warming. As noted by Price [5] over 25 years ago, temperature-based measurements, such as the urban-rural temperature differential, often fail to reflect the magnitude of heat islands created by urban areas of different sizes. 
Price states, "[f]or investigation of urban heating, the peak temperature is less significant than a summation (area integral) of the excess power radiated as a result of the surface temperature elevation" (p. 1557). In other words, a measure of the excess flux of thermal energy from a city provides a more accurate indicator of the total magnitude of warming within an urbanized area than does the urban-rural temperature differential.

In this sense, the magnitude of surface warming between urban and rural zones (defined herein as the excess flux of sensible heat energy) is more relevant to urban planning policy than heat island intensity (the urban - rural temperature differential). The reason for this is that the area-integrated flux of heat energy emitted from a city or parcel provides a more accurate indicator of the volume of the near surface atmosphere influenced by elevated surface temperatures than does the surface temperature differential. In this sense, two cities of similar climate and development type but dissimilar land area are likely to exhibit similar average surface temperatures but different fluxes of energy. As the area of a city expands, the volume of the heat island "dome" - a volume subject to elevated air temperatures and enhanced pollution formation - expands as well. Measures of heat island intensity across cities may not reflect a differential in the volume of heat island domes. While smaller in scale, the same is true when comparing two parcels of different area.

\subsection{Approximating the sensible heat flux}

While aerial and satellite radiometers provide the most viable means of obtaining continuous surface thermal data across a large study region, a principal limitation of remote sensing studies of the urban heat island effect is a failure to account for the variable distribution of surface emissivity. Given the wide array of surface material types found in urban areas, ranging from heavily canopied areas to the impervious materials of driveways and rooftops, radiant flux densities recorded by thermal sensors are prone to systematically underestimate thermodynamic temperatures from the heterogeneous surfaces of urban environments. As a result, the surface radiant flux density must be adjusted to more closely approximate the black body flux density, which is a better indicator of sensible heat.

In summary, to be useful in planning research, an indicator of the urban heat island effect must be spatially compatible with the legal dimensions of land use control, and must accurately approximate the quantity of energy contributed by a single land parcel to elevated temperatures within cities. What follows in the remainder of the paper is an application of this conceptual approach to the Atlanta, Georgia metropolitan region and a presentation of policy insights that may be gleaned from this analysis.

\section{Methods}

In May of 1997, the National Aeronautic and Space Administration (NASA) obtained high resolution multispectral imagery over the Atlanta, Georgia 
metropolitan region for the purpose of investigating the influence of land use on urban heat island formation. Recorded from an aerial platform with the Advanced Thermal and Land Applications Sensor (ATLAS) at a ground resolution of 10 meters, 15 channels of multispectral data were obtained over a $48 \mathrm{~km}^{2}$ region by NASA during the course of two flights and made available to a range of research efforts known collectively as "Project Atlanta." The exceptionally high spatial resolution of this data permits the thermal characteristics of high-density urban parcels to be reliably measured and integrated into a parcel level geographic information system. With the aid of the Project Atlanta data, a measure of the parcel "net black body flux" was derived for every single family residential lot in the Atlanta study region. What follows is a brief overview of the methods employed to derive a measure of the net black body flux based on remotely sensed data; for a full discussion of this methodology, please see Stone and Norman [6].

\subsection{Deriving the net black body flux}

The first step in estimating the parcel net black body flux requires that the thermal characteristics of individual land parcels be measured. To do so, parcel boundary information for the approximately 104,000 single-family parcels located in the study region was obtained from the City of Atlanta and Fulton County offices of tax assessment. By registering the multispectral imagery and parcel geography data layers to the same coordinate system, it was possible to spatially overlay the two datasets in a geographic information system. Once integrated, the average apparent radiant flux density of each parcel was derived through a zonal summary function.

In addition to the apparent radiant flux density, information on the composite parcel emissivity and emitted sky radiation was needed to derive the black body flux density. Employing the method of Carlson et al [7], the fraction of vegetative cover for each land parcel was estimated through the derivation of a normalized difference vegetation index (NDVI) with ATLAS bands in the red and near infra-red spectral regions. Based on published data for vegetative and impervious materials characteristic of residential zones, an emissivity value of 0.96 was assigned to the vegetative component of each parcel and a value of 0.92 was assigned to the impervious component (Jensen [8]; Oke [9]). The composite parcel emissivity was then calculated based on the area of pervious and impervious materials per parcel and used, in concert with the average value of emitted sky radiation for the day of ATLAS data collection, to compute the estimated parcel black body flux density.

As a final step, the quantity of radiant energy attributable to predevelopment land characteristics - the rural "background" radiation - was subtracted from the measured average parcel black body flux density. In deriving an indicator of the urban heat island effect, we are interested in the thermal differential between developed and undeveloped land features. When measuring heat island intensity, this variable is quantified as the temperature differential between developed and undeveloped zones. When measuring heat island magnitude, this variable becomes the flux differential between developed and undeveloped zones. To 
calculate this flux differential at the parcel level, we first calculate the average black body flux density for a developed parcel and then subtract from this quantity an estimate of the predevelopment black body flux density that would result were the same parcel occupied by a natural land cover, such as forest canopy cover. This flux density differential is then multiplied by the parcel area to estimate the parcel net black body flux.

\subsection{Measuring parcel design}

Following the development of the parcel net black body flux measure, four parcel design variables were derived for this study based on city and county property tax records and the multispectral ATLAS imagery. These included the area of impervious materials, such as driveways and building footprints, the area of lawn and landscaping, the proportion of the parcel overlaid by tree canopy, and the number of bedrooms in the residential structure. This final variable was used to control for residential capacity. In short, I am interested in comparing the thermal characteristics of parcels varying in size and material composition but similar in the number of residents the parcel was designed to accommodate. The inclusion of this variable in our analysis ensures that differences in thermal performance by region of the city are not attributable to differences in residential capacity.

Information on the area, footprint size of residential buildings (houses and detached buildings), and number of bedrooms in the residential structure was obtained from the City of Atlanta and Fulton County offices of tax assessment. As driveway areas are not recorded in the property assessment process, a sample of parcels stratified by age and size was selected to estimate the area of these paved surfaces. The driveways of selected parcels were then measured directly to determine how paved areas scale with parcel size throughout the study region. In combination, the estimated area of driveway paving and the area of the building footprint constitute the impervious component of the parcel. The area of lawn and landscaping was derived by subtracting the estimated impervious area from the total parcel area. Finally, the proportion of each parcel overlaid by tree canopy was estimated from the NDVI.

\section{Analysis and results}

With the aid of the Statistical Package for the Social Sciences (SPSS), an analysis was performed to assess the influence of parcel design on the parcel net black body flux. The average single family residential parcel in the database is characterized by a three bedroom house and is approximately $1,360 \mathrm{~m}^{2}$ in area, of which $208 \mathrm{~m}^{2}$ or $15 \%$ is occupied by the impervious components of the house and driveway, with the remaining $85 \%$ occupied by lawn and landscaping. Approximately $45 \%$ of the average single-family residential parcel is overlaid by tree canopy cover.

To evaluate the graphic correlation between parcel area, material composition, and surface warming, values of increasing lot size are plotted 
against impervious area, lawn area, and the net black body flux in Figure 1. As illustrated in this figure, the magnitude of surface warming scales closely with lot size, with the mean net black body flux increasing by a factor of about six between the highest and lowest density classes. While this descriptive evidence supports my hypothesis of a negative relationship between the density of singlefamily residential development and surface warming, it is important to note that this simple covariation does not account for the distribution of residential capacity or tree canopy cover throughout the region. If larger lot sizes are also associated with greater residential capacities (e.g., four and five bedroom houses) and a less mature tree canopy cover, then the relationship between the density of development and thermal performance may be attributable to incompatible design objectives or to the age of development in different areas of the city. In order to control for these important influences, a multivariate statistical model is developed and evaluated in the following section.

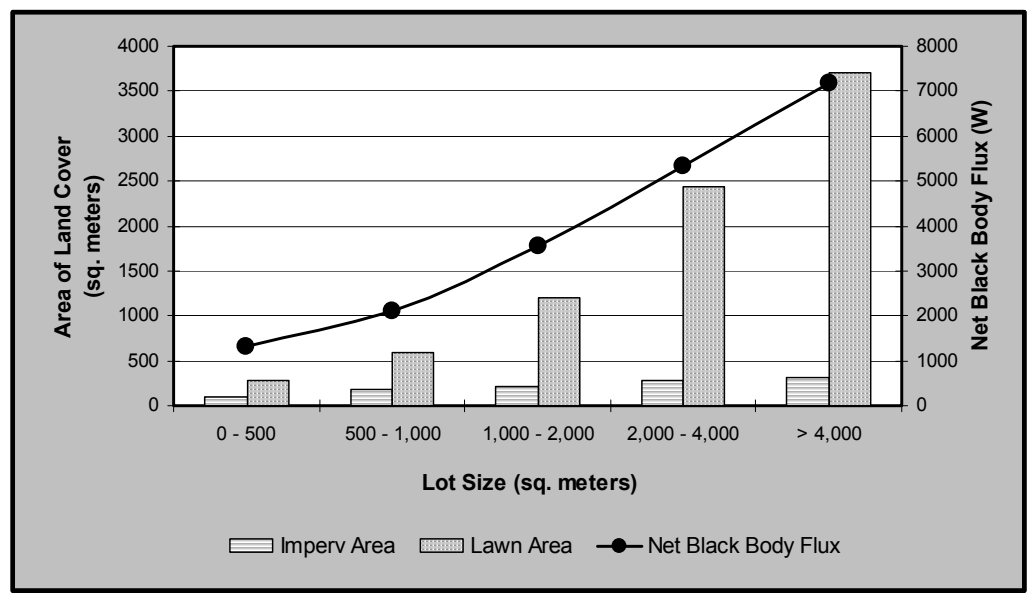

Figure 1: Parcel attributes by lot size.

\subsection{Explanatory analysis}

In order to quantify the potential thermal benefits of specific design based strategies, ordinary least squares was used to develop a predictive model linking parcel design to the net black body flux. Four independent variables were incorporated into the model to explain the net black body flux. These included the area of impervious cover, the area of pervious cover or lawns and landscaping, the percentage of the parcel overlaid by tree canopy, and the number of bedrooms in the residential structure.

With the aid of SPSS, a set of parameter estimates and model summary statistics were generated for the approximately 104,000 single family residential parcels located in the Atlanta study region. The results of this modeling process are presented in Table 1. 
The results of the regression analysis indicate that the various parcel design attributes are significantly related to the parcel net black body flux. As expected, the area of impervious and pervious materials were found to exhibit a significant positive relationship with parcel warming when controlling for other parcel design attributes believed to be associated with the dependent variable. Also as expected, the presence of tree canopy cover was found to significantly decrease the magnitude of warming per parcel. These relationships were found to hold when controlling for the number of bedrooms in the residential structure, suggesting that the elevated surface warming is not solely attributable to variation in residential capacity.

Table 1: Regression analysis results for parcel net black body flux (W)*.

\begin{tabular}{cccc}
\hline Variable & B-Coefficient & $\begin{array}{c}\text { Standardized } \\
\text { Coefficient }\end{array}$ & Significance \\
\hline Impervious Area $\left(\mathrm{m}^{2}\right)$ & 0.079 & 0.274 & $<.001$ \\
Lawn Area $\left(\mathrm{m}^{2}\right)$ & 0.013 & 0.553 & $<.001$ \\
Tree Canopy Cover $(\%)$ & -23.93 & -0.419 & $<.001$ \\
Number of Bedrooms & 0.438 & 0.019 & $<.001$ \\
\hline Summary Statistics & $\begin{array}{c}\text { Adj. R-Square } \\
0.57\end{array}$ & $\begin{array}{c}\text { F-Statistic } \\
35,217\end{array}$ & $\begin{array}{c}\text { F Significance } \\
<.001\end{array}$ \\
\hline
\end{tabular}

* The square root of the net black body flux was used in this model as a variance stabilization transformation.

\section{Discussion and conclusions}

The results of this analysis provide compelling evidence that the size and material composition of single family residential parcels are significantly related to the magnitude of surface warming in the Atlanta study region. Specifically, smaller, higher density parcels were found to be associated with a lower net black body flux than larger, lower density parcels when controlling for the class of land use and the number of bedrooms in the residential structure. While both the area of impervious materials and lawn and landscaping were found to be positively related to the parcel net black body flux, the area of lawn and landscaping - a strong correlate of parcel size - was found to be the strongest predictor of excess surface warming.

In contrast to previous work on the urban heat island (e.g., Hoyano [10]), the results of this study support the hypothesis that lower density, dispersed patterns of urban residential development contribute more surface energy to regional heat island formation than do higher density, compact forms. While specific to the Atlanta metropolitan region, due to the uniformity of building materials and development patterns found throughout North American cities, these findings are 
generalizable to a number of large, mid-latitude cities characterized by a similar climatological and physiographic characteristics. For such cities, this work illuminates at least three specific planning strategies that may be employed to offset the excess surface heat energy emitted from both existing and future residential development, including reductions in the area of residential lawn space, reductions in the area of impervious cover, and an increase in tree canopy cover. Each of these strategies is discussed briefly in turn.

The results of this analysis suggest that the most effective design-oriented strategy for mitigating the thermal impacts of new housing development is a reduction in the area of the residential lawn. For the average parcel, a reduction in the lawn area of $25 \%$, holding other design attributes constant, would be associated with a $14 \%$ reduction in the parcel net black body flux. By serving to increase the density of land use, a reduction in the lawn area of new residential development would offset enhanced surface warming through at least two mechanisms. First, by reducing the average lot size of development at the urban periphery, the area of rural land converted from forest, Atlanta's natural land cover, to urban land uses is reduced, constraining the zone of enhanced warming. Second, by limiting the total zone of urban expansion, higher density development limits the extension of roadways and other thermally intensive infrastructure, also serving to preserve rural landscapes.

The finding of a positive relationship between the area of impervious cover and the parcel net black body flux confirms the well established role of mineralbased building and paving materials in urban heat island formation. The most widely advocated approach to mitigating the thermal impacts of impervious materials is through the application of high albedo surface treatments to increase surface reflectivity - an approach that is cost effective and applicable to both new and existing development. For new construction, a reduction in the total area of impervious cover provides an additional tool for offsetting an increase in surface warming. These findings suggest that a $25 \%$ reduction in the impervious cover of an average single-family parcel would be associated with a $15 \%$ reduction in the net black body flux. When combined with an equivalent reduction in lawn area, parcel warming was found to be $27 \%$ lower.

While the potential to reduce the thermal impacts of new growth are significant, it is important to note that changes to a city's land development regulations will have only a limited influence on existing development. As the vast majority of the Atlanta region's 2020 built area is already in place, changes in future peripheral development will serve to offset continued growth rather than to abate present day heat island formation. In light of this observation, the most effective design-oriented strategies for reducing total regional warming must address both new and existing development. As noted above, one strategy for doing so would entail the replacement of traditional driveways with driveway runners at the time of routine resurfacing. A second critical strategy for existing development entails the planting of trees. As indicated by our analysis, for the average single family parcel, an increase in tree canopy cover from 45 to $60 \%$ reduces the parcel net black body flux by $13 \%$. For trees strategically planted along roadways and in proximity to houses, the thermal benefits are likely to be 
greater. In combination, a $25 \%$ reduction in the areas of pervious and impervious materials, and an increase in parcel tree canopy cover from 45 to $60 \%$, were found to reduce the net black body flux from a parcel of average size by approximately $40 \%$.

\section{References}

[1] Kalnay, E. \& Cai, M., Impact of urbanization and land use change on climate. Nature, 423, 528-531, 2003.

[2] Foley, J., Defries, R., et al, Global consequences of land use. Science, 309, 570-574, 2005.

[3] Roth, M., Oke, T., Emery, W., Satellite-derived urban heat islands from three coastal cities and the utilization of such data in urban climatology. International Journal of Remote Sensing 10, 1699-1720, 1989.

[4] Nichol, J., High-resolution surface temperature patterns related to urban morphology in a tropical city: A satellite-based study. Journal of Applied Meteorology 135, 135-46, 1996.

[5] Price, J., Notes and correspondence: Assessment of the urban heat island effect through the use of satellite data. Monthly Weather Review 107, $1554-1557,1979$.

[6] Stone Jr., B., Norman, J., Land use planning and surface heat island formation: A parcel based radiation flux based approach. Atmospheric Environment, forthcoming. Study methodology reprinted with permission from Elsevier.

[7] Carlson, T., Capehart, W., Gillies, R., A new look at the simplified method for remote sensing of daily evapotranspiration, Remote Sensing of Environment 54, 161-167, 1995.

[8] Jensen, J., Remote sensing of the environment: An earth resource perspective. Prentice Hall, Upper Saddle River, NJ, 2000.

[9] Oke, T., Boundary layer climates, Routledge, New York, NY, 1987.

[10] Hoyano, A., Relationships between the type of residential area and the aspects of surface temperature and solar reflectance (based on digital image analysis using airborne multispectral scanner data). Energy and Buildings 7, 159-73, 1984. 